\title{
RISPERIDONE AND OLANZAPNE IN-PATIENT UTILIZATION IN THE UNIVERSITY OF MALAYA MEDICAL CENTRE, KUALA LUMPUR
}

\author{
Hatim A and Yen TH \\ Department of Psychological Medicine, University of Malaya Medical Centre, Lembah Pantai, 50603 Kuala Lumpur, Malaysia
}

\begin{abstract}
The objective of this study was to compare in-patient drug use patterns, costs, and outcomes associated with risperidone or olanzapine in a naturalistic clinical setting. Retrospective chart reviews of 92 patients with psychotic disorders were conducted at the University of Malaya Medical Centre (UMMC). Data was collected from patients who were hospitalized and for whom risperidone or olanzapine was the drug of first choice for long-term pharmacologic treatment.

Proportion of patients for whom efficacy of the studied treatment could be established (as rated by the treating physician) was higher, but not significantly, with risperidone compared to olanzapine $(p=0.46)$. The average dose of the studied medication was $2.9 \pm 1.0 \mathrm{mg} / \mathrm{day}$ for risperidone and $9.7 \pm 2.4 \mathrm{mg} / \mathrm{day}$ for olanzapine. The total cost was significantly higher $(p<0.0001)$ for olanzapine (RM140.40; 95\% CI: 108.4-181.9) compared to risperidone (RM50.80, 95\% CI: 39.5-65.3). The daily cost was also significantly higher for olanzapine (RM19.16; 95\% CI: 17.72-20.72) compared to risperidone (RM4.95; 95\% CI: 4.46-5.51, $p<0.0001$ ). Analysis of responders showed significantly higher daily and total costs of treatment with olanzapine compared to risperidone. These preliminary data suggest that treatment with risperidone may be more costeffective than treatment with olanzapine. However, a longer duration of study and more data is needed before a proper conclusion on cost-effectiveness is made. (JUMMEC 2006; 9(1): 23-29)
\end{abstract}

KEYWORDS: antipsychotic, outcome

\section{Introduction}

The novel or atypical anti-psychotic medications introduced over the last decade represent a significant step forward in pharmacotherapy. Risperidone (Risperdal ${ }^{\circledR}$ ) and olanzapine $\left(Z y p r e x a^{\circledR}\right)$ are novel anti-psychotic agents belonging to the new class of atypical antipsychotics that was first defined by the introduction of clozapine. The exact mechanism of action of both drugs has not been fully elucidated. It may involve antagonism at serotonin type 2 (and type 3 and 6 for olanzapine) and dopamine receptors (I).

While conventional anti-psychotics effectively reduce psychotic symptoms, they often induce extrapyramidal side effects and tardive dyskinesia. The atypical agents are generally free of these unwanted effects and generally have a more favourable adverse-effect profile than conventional anti-psychotics $(2,3)$. Moreover, in addition to effectively treating positive psychotic symptoms, atypical agents are often helpful for patients unresponsive to conventional agents and may be more effective for negative symptoms and cognitive dysfunction. These characteristics improve drug compliance and yield a decline in the number of relapses and the need of hospitalization $(4,5)$.

While cost-effectiveness of atypical anti-psychotics versus the conventional drugs has been extensively documented and demonstrated, the relative costeffectiveness of the atypical anti-psychotics among each other has not yet been widely investigated. A study from North America suggests that olanzapine is associated with higher treatment costs (on average twice as costly), while this is not compensated for by any clinical advantage (6). The present study was a single centre, retrospective comparative study. The objective of this study was to compare the drug usage pattern, the costs and outcomes associated with

\section{Correspondence:}

Dr. Ahmad Hatim Sulaiman

Department of Psychological Medicine

Faculty of Medicine

University of Malaya

50603 Kuala Lumpur, Malaysia

Email: hatim@um.edu.my 
treatment of psychotic disorders with either risperidone or olanzapine within a single hospital setting in Malaysia.

\section{Methods}

\section{Study outline}

Data was collected at the University of Malaya Medical Centre (Malaysia) from patients who had been hospitalized and for whom risperidone or olanzapine was the first intended drug for long-term pharmacological treatment after admission.

The total number of patients required within each treatment group was 33. The most recent admissions were systematically checked in reverse chronological order and patients were included if the following criteria were met:

I. Risperidone or olanzapine was the first intented drug for long-term treatment;

2. Patient was not older than 65 years; and

3. The patient was discharged from the hospital before 120 days.

The most recent patients treated by olanzapine and the most recent patients treated by risperidone were selected. Data regarding demography, history of psychiatric illness (diagnosis, number of previous hospitalizations), diagnosis upon admission, hospitalization information (length of hospital stay, discharge status), medication history, usage pattern of drugs used over the length of stay in hospital, dose details of the study treatments (risperidone and olanzapine, details of other neuroleptics or other relevant concomitant medications, treatment efficacy (efficacy assessment: effective, ineffective or partially effective as rated by the treating physician) and side effects related to neuroleptics were collected.

\section{Primary parameters (outcome measures)}

The primary parameters were the average daily costs of the drugs (Risperidone/Olanzapine) under study.

\section{Secondary parameters (outcome measures)}

The secondary parameters were the average daily dose and cost of studied treatments (risperidone or olanzapine), the time to discharge, the proportion of patients who discontinued treatment and switched from risperidone or olanzapine to other anti-psychotic treatment, the treatment efficacy as rated by the treating physician, the number of days before efficacy was established and the side effect profile.

\section{Patient populations}

Two populations were considered:

I. The intention-to-treat (ITT) populations, consisting of patients who had been treated with the treatment to which they were assigned;

2. The responders, defined as patients whose treatment was judged as effective by the treating physician and who did not discontinue the treatment for any reason except if the treatment was no longer deemed necessary.

\section{Data analysis}

Dosage parameters were analyzed by descriptive methods (mean, standard deviation, median, minimum and maximum) without statistical comparison between risperidone and olanzapine. Costs between treatments were analyzed by descriptive statistics and compared by using t-test on the log-transformed data because log-normal distribution fits the data better than the normal distribution. The mean cost was summarized by the geometric mean and its $95 \%$ confidence interval.

Proportions of patients were compared between treatments using Fisher's exact test. Number of days before efficacy was established, was compared using the non-parametric Mann-Whitney rank test.

The time to event parameters (e.g., time to onset of efficacy, time to discharge) was also analyzed using survival analysis methods to take into consideration censored data (patient for whom efficacy was not reached). The Kaplan-Meier product-limit estimate of the survival function was calculated and the comparison between treatments was carried out using non-parametric tests (Generalized Wilcoxon test and log-rank test).

\section{Ethics}

The study was approved by the University of Malaya Medical Centre ethics committee. Consent was obtained from patients prior to data collection.

\section{Results}

Ninety-two patients (Risperidone: 43, Olanzapine: 49) were part of the study. There were 67 responders (Risperidone: 35, Olanzapine: 32). Among the 25 nonresponders, the study treatment was rated as ineffective by the physician in 12 patients, the treatment was rated as partially effective in ten others, and the efficacy was not assessed in three patients. 
Demography and patient's profile are summarized in Table I. Patients treated with risperidone were significantly older at admission than those treated by olanzapine (median: 35.9 vs. 29.5 years, respectively, $p=0.02)$. The age at onset of first symptoms was higher in the risperidone group than in the olanzapine group (median: 27.3 vs. 25.5 years, respectively). However, the difference was not statistically significant $(p=0.1 \mathrm{I})$. The proportion of females and males was similar in both groups $(p=0.40)$. The groups differed significantly regarding the pattern of diagnosis $(p=0.002)$. There were proportionally more patients with diagnosis other than schizophrenia in the olanzapine group (bipolar: $33 \%$; other diagnosis: $31 \%$ ) than in the risperidone group (bipolar: 7\%; other diagnosis: 16\%).

Table I. Demography and patient's profile (ITT patients)

\begin{tabular}{|c|c|c|c|c|}
\hline \multicolumn{2}{|c|}{ Parameter } & Risperidone $(\mathrm{N}=43)$ & Olanzapine (N=49) & $p$ \\
\hline \multicolumn{2}{|c|}{ Age at onset of first symptoms (y) } & $31.3 \pm 14.9$ & $27.2 \pm 9.0$ & 0.11 \\
\hline \multicolumn{2}{|c|}{ Age at admission $(y)$} & $39.1 \pm 16.4$ & $32.2 \pm 11.3$ & 0.02 \\
\hline \multirow[t]{2}{*}{ Gender } & Males & $18(43 \%)$ & $26(53 \%)$ & 0.40 \\
\hline & Females & $24(57 \%)$ & $23(47 \%)$ & \\
\hline \multicolumn{2}{|c|}{ Diagnosis: } & & & 0.002 \\
\hline & - Catatonic schizophrenia & I (2\%) & 0 & \\
\hline & - Disorganised schizophrenia & I (2\%) & 0 & \\
\hline & - Paranoid schizophrenia & $23(53 \%)$ & 14 (29\%) & \\
\hline & - Undifferentiated schizophrenia & $8(19 \%)$ & $4(8 \%)$ & \\
\hline & - Bipolar & $3(7 \%)$ & $16(33 \%)$ & \\
\hline & - Other diagnosis & $7(16 \%)$ & $15(3 \mid \%)$ & \\
\hline \multicolumn{2}{|c|}{ Previous hospitalization } & $22(51 \%)$ & 27 (55\%) & 0.83 \\
\hline \multicolumn{3}{|c|}{ Number of previous hospitalizations: } & & 0.45 \\
\hline & 0 & $21(49 \%)$ & $22(45 \%)$ & \\
\hline & $1-5$ & $19(44 \%)$ & $25(51 \%)$ & \\
\hline & $6-10$ & $3(7 \%)$ & $2(4 \%)$ & \\
\hline \multicolumn{2}{|c|}{ History of medication available } & $26(60 \%)$ & $27(55 \%)$ & 0.68 \\
\hline \multicolumn{2}{|c|}{$\begin{array}{l}\text { Use of anti-psychotics during } \\
\text { the previous year }(\ddagger)\end{array}$} & $26(100 \%)$ & $27(100 \%)$ & - \\
\hline \multicolumn{3}{|c|}{ Number of previous anti-psychotics: $(\ddagger)$} & & 0.21 \\
\hline & 0 & 0 & 0 & \\
\hline & $\mathrm{I}$ & $12(46 \%)$ & $9(33 \%)$ & \\
\hline & 2 & $13(50 \%)$ & 14 (52\%) & \\
\hline & 3 & I (4\%) & $4(15 \%)$ & \\
\hline \multicolumn{5}{|c|}{$\begin{array}{l}\text { Number of patients who discontinued } \\
\text { previous anti-psychotics: }(\S)\end{array}$} \\
\hline & - for any reason & $26(100 \%)$ & $26(96 \%)$ & $>0.99$ \\
\hline & - for lack of efficacy & $17(65 \%)$ & $19(70 \%)$ & 0.77 \\
\hline & - for side effects & $12(46 \%)$ & $12(44 \%)$ & $>0.99$ \\
\hline & - for other reason(s) & $4(15 \%)$ & $5(19 \%)$ & $>0.99$ \\
\hline \multicolumn{4}{|c|}{$\begin{array}{l}\text { Number of previous anti-psychotics } \\
\text { discontinued: }(\ddagger)\end{array}$} & 0.7 \\
\hline & 0 & 0 & I (4\%) & \\
\hline & I & $12(46 \%)$ & II (4I\%) & \\
\hline & 2 & $13(50 \%)$ & II (4I\%) & \\
\hline & 3 & I (4\%) & $4(15 \%)$ & \\
\hline
\end{tabular}

Values are numbers of patients (\%) except for age: mean $\pm S D$

$(\ddagger)$ : Percentages are calculated using the patients with available history of medications as denominator ( $N=26$ and 27 patients for risperidone and olanzapine, respectively);

$(\S)$ : Percentages are calculated using the patients who took previous anti-psychotics ( $N=26$ and 27 patients for risperidone and olanzapine, respectively). 
The proportion of patients with previous hospitalizations was similar in both groups $(p=0.83)$. The number of previous hospitalizations exceeded five times in $7 \%$ of the risperidone patients and in $4 \%$ of the olanzapine patients. Of the patients with available history of medication, there were $60 \%$ in the risperidone group and $55 \%$ in the olanzapine group $(p=0.68)$. Of these patients, all in both groups had used anti-psychotics in the previous year. The number of previous anti-psychotics used by patients was similar in both groups $(p=0.21)$. The proportion of patients who discontinued previous anti-psychotics was also similar in both groups. Reasons for discontinuation were lack of efficacy in 36 patients (Risperidone: 17; Olanzapine: 19), side effects in 24 patients ( 12 in each group), and other reasons in nine patients (Risperidone: 4; Olanzapine: 5). Based on the list of previous anti-psychotics, only one olanzapine patient was already treated with the medication under study when admitted at the hospital.

Main parameters of interest are summarized in the Table 2. Proportion of patients for whom efficacy of the treatment could be established (as rated by the treating physician) was higher with risperidone (8I\%) than with olanzapine (70\%). However, the difference was not statistically significant $(p=0.46)$. The number of days before treatment efficacy was established was similar in both groups (median: 6.0 days for both treatments, $p=0.60$ ). The difference between groups in the curve of the time to efficacy distribution function (including censored observations) was not statistically significant ( $p \geq 0.59$, Figure I).

The average daily dose was $2.9 \mathrm{mg} /$ day for risperidone patients and $9.7 \mathrm{mg} /$ day for olanzapine patients. The study treatment duration was slightly longer in the risperidone group than in the olanzapine group ( 10.2 vs. 7.3 days, respectively). However, the difference reached a statistical tendency $(p=0.07)$. The average daily cost of the medication was significantly higher for olanzapine than for risperidone (Ringgit Malaysia (RM) 19.16 vs. RM4.95, respectively, $p<0.000$ I). The total cost of treatment was significantly higher for olanzapine than for risperidone (RMI40.4 vs. RM50.8, respectively, $p<0.000 \mathrm{I})$. When considering the responders only (Table 3), the results also showed significantly higher daily and total costs of treatment with olanzapine compared to risperidone.

Utilization of other neuroleptics and other relevant concomitant medications (anti-epileptics, anti-Parkinson, anxiolytics, hypnotics and sedatives, and anti-histamines) is summarized in Table 4. The proportion of patients who took at least one other neuroleptic or one other relevant concomitant medications (started at any time)

Table 2. Main parameters of interest (ITT patients)

\begin{tabular}{|c|c|c|c|}
\hline Parameter & Risperidone $(\mathrm{N}=43)$ & Olanzapine $(\mathrm{N}=49)$ & $p$ \\
\hline Number of patients & $43(100 \%)$ & $49(100 \%)$ & - \\
\hline Number of patients with effective treatment $(\S)$ & $35(81 \%)$ & $32(70 \%)$ & 0.46 \\
\hline Number of days before efficacy was established $(\ddagger)$ & $6(|-2|)$ & $6(2-14)$ & 0.60 \\
\hline Number of patients who discontinued the treatment & 0 & I (2\%) & $>0.99$ \\
\hline Average daily dose (mg) & $2.9 \pm 1.0$ & $9.7 \pm 2.4$ & \\
\hline Study treatment duration (days) & $10.2(8.4 ; 12.5)$ & $7.3(5.9 ; 9.1)$ & 0.07 \\
\hline Total cost (RM) of treatment drug & $50.8(39.5 ; 65.3)$ & | 40.4 ( $108.4 ; \mid 81.9)$ & $<0.0001$ \\
\hline Daily cost (RM) of treatment drug & $4.95(4.46 ; 5.5 I)$ & $19.16(17.72 ; 20.72)$ & $<0.000$ I \\
\hline
\end{tabular}

Values are patient numbers (\%) except for dose: mean $\pm S D$, number of days before efficacy: median (minimum - maximum) and costs and study treatment duration: geometric means ( $95 \%$ confidence interval).

(§): Efficacy of three olanzapine patients was not assessed ( $N=43$ for risperidone, $N=46$ for olanzapine);

$(\ddagger)$ : Statistics are calculated on the patients whose efficacy was established ( $N=35$ for risperidone, $N=32$ for olanzapine).

US\$ $=$ RM3.80

Table 3. Main parameters for responders

\begin{tabular}{|c|c|c|c|}
\hline Parameter & Risperidone (N=35) & Olanzapine $(\mathrm{N}=32)$ & $p$ \\
\hline Average daily dose (mg) & $2.9 \pm 0.9$ & $9.5 \pm 1.9$ & \\
\hline Study treatment duration (days) & II.3 (9.I; I4.I) & $10.2(8.4 ; 12.5)$ & 0.58 \\
\hline Total cost (RM) of treatment drug & $56.7(43.8 ; 73.5)$ & $195.2(150.5 ; 253.2)$ & $<0.0001$ \\
\hline Daily cost (RM) of treatment drug & $5.0 \mathrm{I}(4.50 ; 5.58)$ & $19.09(17.48 ; 20.85)$ & $<0.0001$ \\
\hline
\end{tabular}

Values are geometric means ( $95 \%$ confidence interval) except for dose: mean \pm SD. 
Table 4. Use of other neuroleptics and other concomitant medications

\begin{tabular}{lccc}
\hline Parameter & Risperidone (N=43) & Olanzapine (N=49) & $p(*)$ \\
\hline $\begin{array}{l}\text { Number of patients who took other neuroleptics } \\
\text { Pre-existing of Ris./Ola. treatment }\end{array}$ & $25(58 \%)$ & $30(61 \%)$ & 0.83 \\
$\begin{array}{l}\text { Since initiation of Ris./Ola. treatment } \\
\text { After initiation of Ris./Ola. treatment }\end{array}$ & $7(16 \%)$ & $4(8 \%)$ & 0.34 \\
\hline $\begin{array}{l}\text { Number of patients who took other relevant } \\
\text { concomitant medications }\end{array}$ & $1(2 \%)$ & & $>0.99$ \\
$\begin{array}{l}\text { Pre-existing of Ris./Ola. treatment } \\
\text { Since initiation of Ris./Ola. treatment }\end{array}$ & $21(49 \%)$ & $19(39 \%)$ & 0.40 \\
After initiation of Ris./Ola. treatment & $4(9 \%)$ & $10(20 \%)$ & $>0.99$ \\
\hline $\begin{array}{l}\text { Number of patients who took other neuroleptics } \\
\text { or concomitant medications }\end{array}$ & $6(12 \%)$ & 0.75 \\
$\begin{array}{l}\text { Pre-existing of Ris./Ola. treatment } \\
\text { Since initiation of Ris./Ola. Treatment }\end{array}$ & $31(72 \%)$ & & \\
After initiation of Ris./Ola. Treatment & $13(30 \%)$ & $32(65 \%)$ & 0.51 \\
\hline
\end{tabular}

$(*)$ : Probability associated with no difference between treatments (Fisher's exact test).

Table 5. Characteristics of the hospitalization (responders)

\begin{tabular}{|c|c|c|c|}
\hline Parameter & Risperidone $(\mathrm{N}=35)$ & Olanzapine $(\mathrm{N}=32)$ & $p(*)$ \\
\hline Total duration (days) & $18.3 \pm 14.5$ & $15.2 \pm 8.2$ & 0.66 \\
\hline Duration of studied treatment (days) & $14.0 \pm 10.6$ & $11.9 \pm 6.9$ & 0.58 \\
\hline $\begin{array}{l}\text { Time (days) between admission } \\
\text { and start of treatment }\end{array}$ & $\begin{array}{l}4.4 \pm 11.0 \\
(0 ; 1.0 ; 64)\end{array}$ & $\begin{array}{l}3.3 \pm 4.0 \\
(0 ; 1.5 ; 15)\end{array}$ & 0.60 \\
\hline $\begin{array}{l}\text { Length of stay in each ward (days) } \\
\text { - Psychiatric }\end{array}$ & $18.3 \pm 14.5$ & $14.3 \pm 8.4$ & 0.40 \\
\hline $\begin{array}{l}\text { Discharge } \\
\text { Number of patients discharged } \\
\text { before or at day } 120\end{array}$ & $35(100 \%)$ & $32(100 \%)$ & - \\
\hline $\begin{array}{l}\text { N patients discharged vs. location: } \\
\text { - Another hospital } \\
\text { - To own home } \\
\text { - Other }\end{array}$ & $\begin{array}{c}2(6 \%) \\
32(91 \%) \\
\quad 1(3 \%)\end{array}$ & $\begin{array}{c}\text { I ( }(3 \%) \\
3 \text { I ( }(97 \%) \\
0\end{array}$ & $>0.99$ \\
\hline Time to discharge: & & & \\
\hline $\begin{array}{l}\text { Time estimate }(95 \% \mathrm{Cl}) \text { for discharge of } \\
25 \% \text { of patients } \\
50 \% \text { of patients } \\
75 \% \text { of patients }\end{array}$ & $\begin{array}{l}9.0(8 ; 12) \\
13.0(11 ; 20) \\
21.0(15 ; 28)\end{array}$ & $\begin{array}{l}9.5(8 ; 12) \\
13.5(10 ; 16) \\
19.5(15 ; 22)\end{array}$ & $\begin{array}{l}0.41(1) \\
0.66(2)\end{array}$ \\
\hline
\end{tabular}

(*): Probability associated with the hypothesis of no difference between treatments (t-test for weight, Mann-Whitney rank test for duration and lengths of stay, Fisher's exact test for number of patient discharged, and log-rank test ( $I$ ) and Wilcoxon test (2) for survival analysis of time to discharge).

was similar with both treatment groups ( 93 vs. $90 \%$ for risperidone and olanzapine, respectively, $p=0.72)$. The proportion of patients who started at least one other neuroleptic or one other concomitant medication at different times was also similar in both groups. When considered separately, the two treatment groups were not significantly different with regards to the propor- tion of patients who started on other neuroleptics or other relevant concomitant medications.

Table 5 shows the characteristics of hospitalization of the responders. Among responders, the total duration of hospitalization was longer in the risperidone group than in the olanzapine group (mean: I 8.3 vs. 15.2 days, 


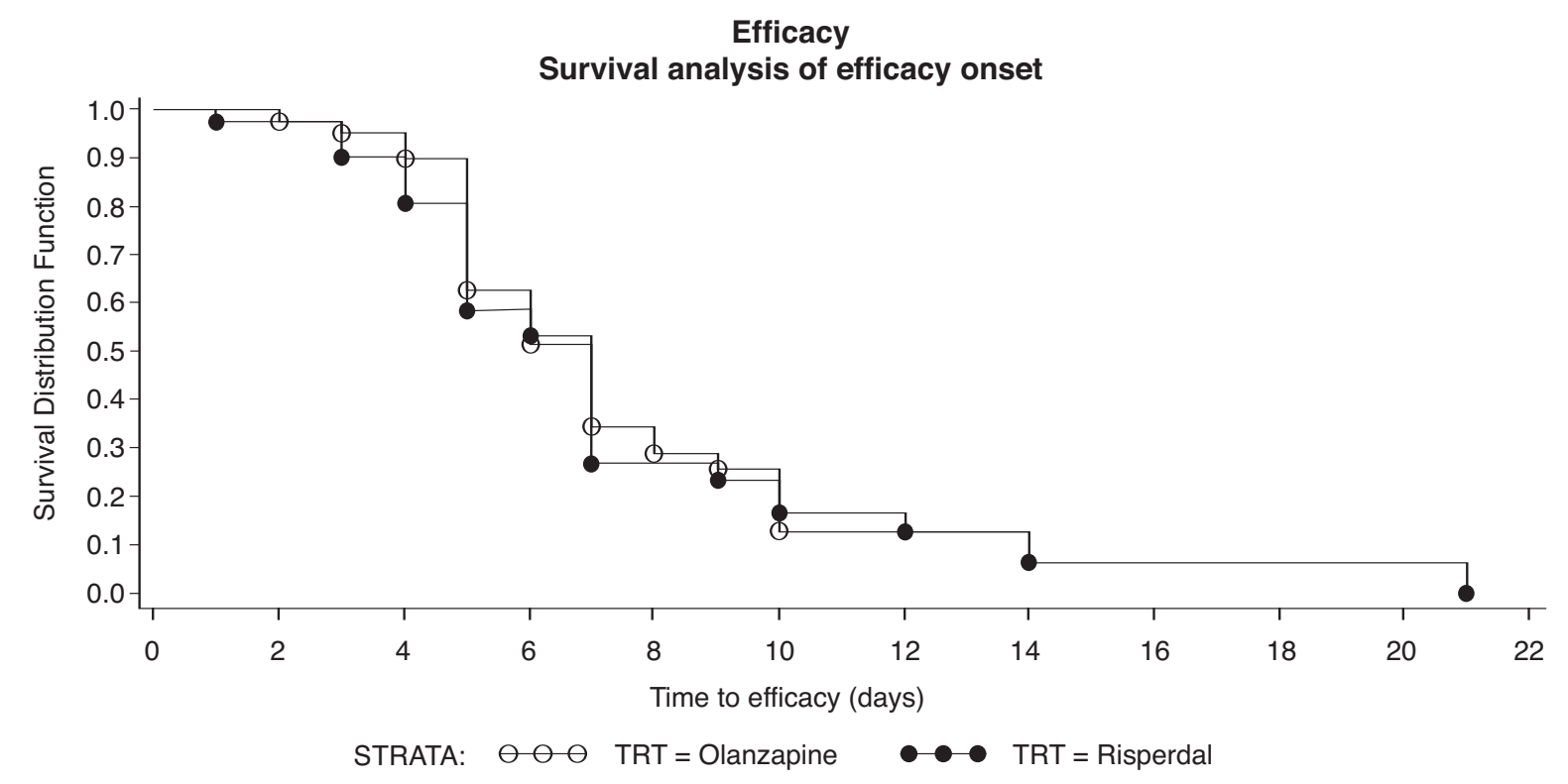

Figure I. Time distribution function of the time (days) to onset of efficacy

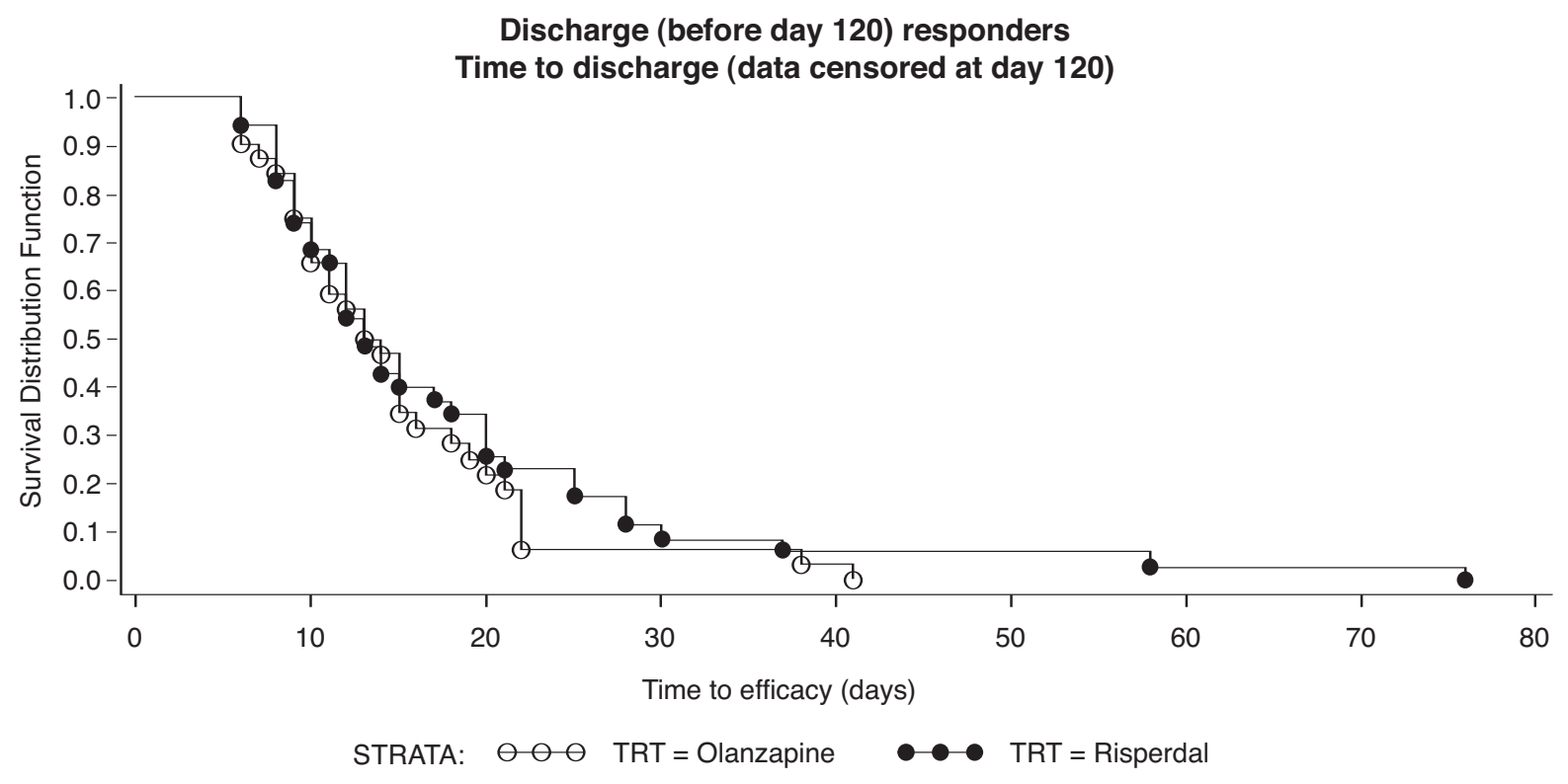

Figure 2. Time distribution function of the time to discharge of responders

respectively). The difference was not statistically significant $(p=0.66)$. This pattern was also true in the actual treatment; duration of risperidone was longer than olanzapine (mean: 14.0 vs. II.9 days, respectively). The difference was also not statistically significant $(p=0.58)$. In the time between admission and start of treatment, no statistical difference between groups was found (mean: 4.4 vs. 3.3 day for risperidone and olanzapine, respectively, $p=0.60$ ).

As observed in the ITT population, all responders in both groups were discharged before 120 days. Most patients were discharged to their own home $(91 \%$ and $97 \%$ for risperidone and olanzapine, respectively). The 
estimate median time to discharge of patients was similar in both treatment groups (I 3.0 vs. I 3.5 days for risperidone and olanzapine, respectively). Moreover, the difference between study groups in the curve of the time to discharge distribution function was not statistically significant $(p \geq 0.40)$ (Figure 2 ).

\section{Discussion}

An episode of psychosis is usually managed through hospitalization and use of anti-psychotic therapies. There is no evidence that really shows one atypical anti-psychotic having better efficacy than the other (7). Therefore, a pharmacoeconomic assessment would primarily be a comparison of the direct costs associated with their administration. The most important driver of costs is probably the drug acquisition costs, which are determined by the actually used dosage schedules of the anti-psychotics and by the need for concomitant medication. Another driver of costs may be the length of hospitalization.

Within this cohort of patients, treatment with risperidone was associated with significantly lower cost compared with olanzapine with no compensation in efficacy and safety. The data suggests that treatment with risperidone may be more cost-effective than treatment with olanzapine. However, there is a clear need to confirm these findings with further retrospective studies in other patient groups and with prospective, randomized, naturalistic studies that would accurately reproduce the clinical conditions in which these agents are used.

Our study has several limitations that must be taken into consideration. By definition, our retrospective study's most obvious limitation is that it is unmasked and unrandomized. The retrospective study design has the potential to compare incomparable groups through some sort of treatment allocation bias. If such a bias was in operation, we would expect to see noticeable differences in patient profiles between treatment groups that would predict the superiority of one treatment over the other. In this study, there is a significant difference in the age of admission that definitely needs consideration. The earlier the age of contact, the more likely it is to have a poor prognosis. There also has been significant difference in the diagnosis. This could affect the conclusions.

\section{Conclusion}

The duration of the study is too short for any significant conclusion on cost-effectiveness. A longer duration would have been more appropriate to see actual effects and side effects that might occur. Despite these limitations, the retrospective study does have the advantage of collecting real-world data beyond the artificial framework that a randomized clinical trial may impose.

\section{Acknowledgement}

This study was supported by a grant from Janssen Cilag, a division of Johnson \& Johnson Sdn. Bhd. The authors would like to thank Dr. Subash Kumar and Dr.Andrew Mohanraj for their assistance in collecting data.

\section{References}

I. Stahl SM. Essential Psychopharmacology: Neuroscientific basis and practical applications. 2nd Edition. Cambridge University Press, 2000; 40 I-458.

2. Tollefson G, Beaseley Jr. C, et al. Olanzapine versus haloperidol in the treatment of schizophrenia and schizoaffective and schizophreniform disorders: Results of an international collaborative trial. Am J Psychiatry 1997; 154: 457-465.

3. Chouinard G, Jones B, Remington G, et al. A Canadian multicenter placebo-controlled study of fixed doses of risperidone and haloperidol in the treatment of chronic schizophrenia patients. J Clin Psychopharmacol, 1993; 13: 25-40.

4. Davies A. Risperidone versus haloperidol: Costeffectiveness. Clin Ther, 1998; 20(I): 196-213.

5. Foster RH, Goa KL. Risperidone: A pharmacoeconomic review of its use in schizophrenia. Pharmacoeconomics, 1998; I4(I): 97-133.

6. Procyshyn M, Zerjav S. Drug utilization patterns and outcomes associated with in hospital treatment with risperidone or olanzapine. Clin Ther, 1998; 20: 12031217

7. Geddes J, Freemantle N, Harrison P, et al. Atypical antipsychotics in the treatment of schizophrenia: Systemic overview and meta-regression analysis. BMJ, 2000; 321: |37|-|376. 